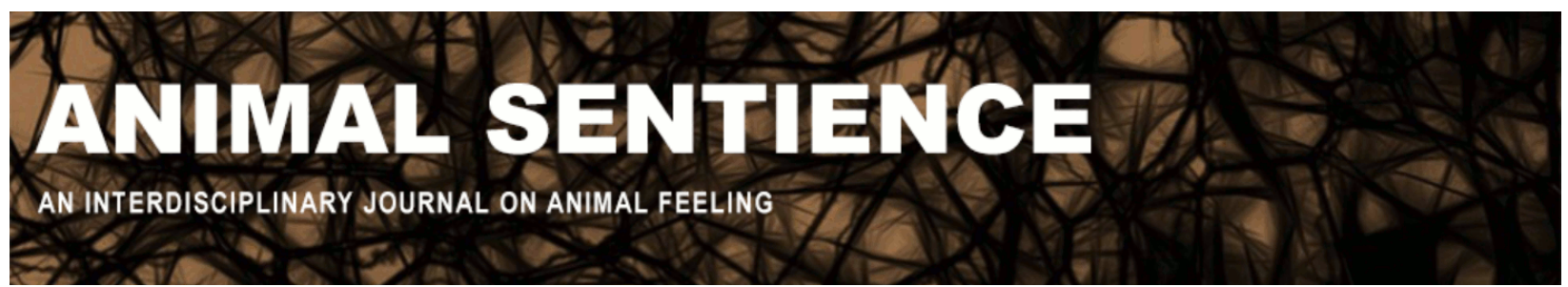

Kendrick, Keith M. (2019) Social cognition in sheep: Welfare implications. Animal Sentience 25(40)

DOI: $10.51291 / 2377-7478.1496$

Date of submission: 2019-06-25

Date of acceptance: 2019-07-04 (c) 


\title{
Social cognition in sheep: Welfare implications
}

Commentary on Marino \& Merskin on Sheep Complexity

\author{
Keith M. Kendrick \\ School of Life Science and Technology \\ University of Electronic Science and Technology of China
}

\begin{abstract}
More research has been carried out on social cognition in sheep than in other farm animal species. Although this has often been featured widely in the media, there is still limited public awareness of it. Marino \& Merskin's review is therefore both important and timely. In my commentary, I focus primarily on what has been established about the complexity of sheep social cognition, at the level of both brain and behavior, and on some of these findings for sheep welfare.
\end{abstract}

Keith Kendrick is professor of social neuroscience currently studying social dysfunction in autism at the University of Electronic Science and Technology of China. Previously he spent over 20 years at the Babraham Institute in Cambridge studying sheep social cognition and demonstrated that they recognize each other visually from facial cues using specialized brain circuitry similar to

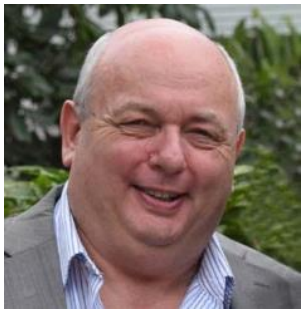
that of humans. Website

When I first became involved in studying social cognition in sheep in the mid 1980's, experiments had demonstrated that they could recognize odor cues from individuals (Baldwin \& Meese, 1977) and that ewes learn to recognize their lambs initially using odor cues (see Sanchez-Andrade \& Kendrick, 2009) and vocalizations (Shillito-Walser, 1978). Despite limited eye movement, sheep - in marked contrast to dogs, for example - have very good visual acuity in their frontal eye fields. We therefore reasoned that sheep might, like humans, use visual cues to recognize important social features of their environment as well as nonsocial features such as food.

Over the subsequent 20 years, using a combination of behavioral and brain-based measures, we showed that sheep not only have the ability to identify individual sheep and humans visually using facial cues but that they do so using specialized neural circuitry previously thought to have evolved only in primates as an adaptation to the increased importance and complexity of their social environment (Tate et al., 2006; Kendrick \& Feng, 2012). With this specialized circuitry, sheep can learn to recognize new individuals relatively quickly and to remember the identify of large numbers of them (probably $>50$ in most cases) for at least several years (Kendrick et al., 2001).

Sheep have a concept of another individual, invariant across perspectives and situations. They can recognize the same familiar conspecific or human from different viewing angles (e.g., frontally and in profile) without additional training and can to some extent recognize younger versions of the same individual (Ferreira et al., 2004; Kendrick, Leigh \& Peirce, 2001; Knolle et al., 2018). What this research demonstrates is that sheep make sophisticated use of facial cues for 
social recognition during the course of their normal lives - not just that they can be trained to do so by various means. Many different species, including insects and birds, can learn to discriminate visual cues from pictures of faces, but this does not imply that they have evolved this ability to recognize and interact with individuals in their social environment (Kendrick \& Feng, 2011). The importance of faces for sheep during their normal lives is further supported by the observation that psychological stress caused by social isolation can be reduced if they are able to view pictures of the faces of other sheep (social buffering); pictures of other species or objects do not have this effect (Da Costa et al., 2004). Providing isolated animals with a mirror can also be effective in this respect: the animals clearly consider the image in the mirror as an individual other than themselves (which in turn confirms that their mirror self-recognition ability is limited, as Marino \& Merskin note). Sheep can also recognize positive and negative emotional cues from the faces of other sheep as well as from some human face expressions - again without the need of training (Tate et al., 2006; Kendrick \& Feng, 2011).

This all suggests that - like humans and other primates - sheep evolved specialized brain circuitry for the visual recognition of individuals and their emotions in their social environment because it increased their chances of survival. It follows that sheep must have a far more complex social environment than many would suppose from just observing their behavior in large-scale flocks. In relation to sheep welfare, it also follows that husbandry practices that result in impaired vision (excessive fleece or horn growth, untreated eye disease, etc.) or an unstable or difficult or impoverished social environment (constantly changing flock members, keeping them in flock sizes which are too large to maintain an effective social network or keeping individuals in social isolation, etc.) are likely to have a negative impact.

Marino and Merksin's review shows that sheep have good learning and memory abilities in a number of contexts and are capable of higher-order rule learning strategies. Rather than entering into further discussion of how intelligent sheep may be in comparison to humans or other species, a more useful consideration for their welfare is how they make use of social information: how they can learn from others, know what others are experiencing (theory of mind), and can even "think" about them in their absence. Lambs learn from their mothers in the context of a strong social bond formed between them that has a life-long influence on both social and sexual preferences (Kendrick et al., 1998).

Sheep have both buddies and enemies in their social environment, and such relationships can be either short- or long-term. Thus, the ability to recognize other individuals accurately is essential. Female sheep also exhibit preferences for approaching specific males when they are sexually receptive, using visual cues from their faces, indicating that facial attractiveness may influence mate choice (Fabre-Nys et al., 1997). Approach and avoidance behaviors in a social environment are influenced by the emotional cues another individual is exhibiting, particularly negative cues associated with fear or anger. Given a choice between approaching pictures of a calm or a stressed/fearful version of the face of the same individual, sheep will approach the former and avoid the latter, with negative emotional cues primarily being displayed through altered appearance of the eyes (more white displayed during fear) and positioning of the ears (flattened when individuals are stressed; Tate et al., 2006). In the context of welfare, the same difference in approach and avoidance behavior can be observed with smiling and angry human faces. The ability to discriminate emotional cues from the faces of others must be largely learned 
from experience and perhaps implies some degree of knowledge about what another individual is experiencing (although this does not necessarily indicate evidence for emotional empathy).

As noted, sheep can remember other familiar sheep and humans for at least several years, displaying emotional responses when reunited. This is perhaps not so remarkable, given similar observations in companion animal species. A more challenging question concerns whether sheep can "think" about such individuals in their absence. We have found some evidence of this in brain activation patterns using nonvisual cues (Kendrick, Leigh \& Peirce 2001). It is still not known whether sheep can voluntarily form visual images without such cueing, but I think it is very likely that for sheep "out of sight" does not mean "out of mind."

In summary, there is plenty of evidence that sheep can have a rich and complex social environment. This has been largely ignored over the years. Marino \& Merskin are to be congratulated for bringing this and other aspects of sheep's cognitive abilities into the spotlight and promoting a better-informed consideration of their welfare needs.

\section{References}

Baldwin, B. A., \& Meese, G. B. (1977). The ability of sheep to distinguish between conspecifics by means of olfaction. Physiology and Behavior 18(5), 803-808.

Da Costa, A. P. C., Leigh, A. E., Man, M. -S., \& Kendrick, K. M. (2004). Face pictures reduce behavioural, autonomic, endocrine and neural indices of stress and fear in sheep. Proceedings of the Royal Society B 271, 2077-2084.

Fabre-Nys, C., Ohkura, S., \& Kendrick, K. M. (1997). Male faces and odours evoke differential patterns of neurochemical release in the mediobasal hypothalamus of the ewe during oestrus: An insight into sexual motivation? European Journal of Neuroscience 9, 1666-1677.

Ferreira, G., Keller, M., Saint-Dizier, H., Perrin, G., \& Lévy, F. (2004). Transfer between views of conspecific faces at different ages or in different orientations by sheep. Behavioural Processes 67(3), 491-499.

Kendrick, K. M., \& Feng, J. (2011). Neural encoding principles in face perception revealed using nonprimate models. In: Handbook of Face Perception (eds. A. J. Calder, G. Rhodes, J. V. Haxby, \& M. H. Johnson), pp. 675-690. Oxford, UK: Oxford University Press.

Kendrick, K. M., da Costa, A. P., Leigh, A. E., Hinton, M. R., \& Peirce, J. W. (2001). Sheep don't forget a face. Nature 414, 165-166.

Kendrick, K. M., Hinton, M. R., Atkins, K., Haupt, M. A., \& Skinner, J. D. (1998). Mothers determine sexual preferences. Nature 395, 229-230.

Kendrick, K. M., Leigh, A. E., \& Peirce, J. (2001). Behavioural and neural correlates of mental imagery in sheep using face recognition paradigms. Animal Welfare 10, S89-101.

Knolle F., Goncalves, R. P., \& Morton, A. J. (2017). Sheep recognize familiar and unfamiliar human faces from two-dimensional images. Royal Society Open Science 4, 1-11.

Marino, L., \& Merskin, D. (2019). Intelligence, complexity, and individuality in sheep. Animal Sentience 25(1).

Sanchez-Andrade, G., \& Kendrick, K. M. (2009). The main olfactory system and social learning in mammals. Behavioural Brain Research 200(2), 323-335.

Shillito-Walser, E. R. (1978). A comparison of the role of vision and hearing in ewes finding their own lambs. Applied Animal Ethology 4, 71-79.

Tate, A. J., Fischer, H., Leigh, A. E., \& Kendrick, K. M. (2006). Behavioural and neurophysiological evidence for face identity and face emotion processing in animals. Philosophical Transactions of the Royal Society of London B: Biological Sciences 361, 2155-2172. 